\title{
DO VISUAL STIMULI AID SELF-GUIDED EMOTIONAL REGULATION? A REVIEW OF CURRENT LITERATURE
}

\author{
Navya Anand ${ }^{1}, \underline{\text { Ramya Mohan }}{ }^{2}$
}

${ }^{1}$ Oxford University, Child \& Adolescent Psychiatry, Oxford, UK

2 National Health Service, UK and Telerad RxDx Healthcare, Child \& Adolescent Psychiatry, India

\section{OBJECTIVES}

Our systematic literature review assessed current evidence for visual stimuli in emotional processing (Standalone visual components / visual cues in relation to other sensory stimuli like music.

\section{BACKGROUND}

This supplements our systematic literature review (2016), exploring the role of music and other therapeutic techniques in self-guided emotional regulation.

From research, musical (not visual) components appear to be the strongest contributors to emotional regulation. When presented with both, four year olds showed a preference for auditory stimuli. However, increased attention to one sensory modality results in increased attention to accompanying sensory information and improved cognitive / behavioural responses in animals / children . Affective responses to emotionally charged stilmuli are magnified when attended to.

Mixed findings around colour-sound associations suggest that human brains adapt to / learn from cross-modal associations / the environment.

Research has established that increased attention results in improved cognitive and behavioural responses, in animals and in children. Increased attention to one sensory modality results in increased attention to other accompanying sensory information. Affective responses to emotionally charged stimuli are magnified when they are attended to.

\section{MATERIALS AND METHODS}

We identified 52 relevant papers ( JSTOR, OVID Medline, Cochrane, PubMed, Science Direct, Taylor \& Francis, Wiley ), including two quantitative neuroimaging ( PET) studies and excluding non-English papers / full qualitative analyses.

\section{RESULTS AND CONCLUSIONS}

Music-colour synaesthesia studies support lifetime consistency ( $92.7 \%$ vs $37 \%$ in controls) and colour-sound associations in musicians ( $n=$ all 250 ) and non-musicians $(n=124 / 600)$. $R$ CBF neural activity differences (Word hearing and activation of identified colour perception regions in human brains ) are more in synaesthetics than controls ( PET ).

Results highlight reliable learned associations between emotions, music and colour from early life (Media influences: TV /Film ) . Sound - colour pairings can elicit reliable responses in the majority. Reliable evidence supports shape with colourbased visual stimuli representing emotional aspects of music . Visual cues enhance attention to musical listening / augment impact of musical components in learnt, self -guided emotional processing , across age and cultures.

Synergestic visual stimuli augmenting emotional processing/ regulation using a self-guided, music -based, multi-modal technique can help ( In anxiety, depression, trauma , adjustment and emotional regulatory conditions ). Focusing on aesthetic / attentional / emotional value (rather than musical structure ) and flexibility in allowing classical / other musical genres to be visualised using this model can increase applicability. Further studies are needed to evaluate.

\section{KEYWORDS}

Emotions , Arts , Creative Arts , Mental Health , Music 